\title{
Differences in levels of inflammatory mediators in meniscal and synovial tissue of patients with meniscal lesions
}

\author{
Takahiro Ogura ${ }^{1 *}$, Miyako Suzuki ${ }^{2}$, Yoshihiro Sakuma², Kazuyo Yamauchi², Sumihisa Orita², Masayuki Miyagi², \\ Tetsuhiro Ishikawa², Hiroto Kamoda², Yasuhiro Oikawa², Izumi Kanisawa', Kenji Takahashi', Hiroki Sakai', \\ Tomonori Nagamine ${ }^{1}$, Hideaki Fukuda ${ }^{1}$, Kazuhisa Takahashi ${ }^{2}$, Seiji Ohtori ${ }^{2}$ and Akihiro Tsuchiya ${ }^{1}$
}

\begin{abstract}
Background: Meniscal injuries are a risk factor for osteoarthritis $(\mathrm{OA})$. While a mechanical pathway between meniscal injury and OA has been described, the biological effects of inflammation on this pathway have yet to be clarified. The aim of our study was to compare levels of specific inflammatory mediators, tumor necrosis factor-alpha (TNF-a), interleukin-6 (IL-6), and nerve growth factor (NGF), in injured and uninjured meniscal tissue and related knee joint synovium.

Methods: Tissue samples were obtained from 19 patients, $31.1 \pm 13.6$ years old, who underwent arthroscopic partial meniscectomy. For analysis, tissue samples were categorized into the following groups: injured meniscal site (IM), non-injured meniscal site (NIM), synovium 'nearest' the lesion (NS), and synovium from the opposite knee compartment, 'farthest' synovium (FS). Levels of inflammatory mediators were determined using enzyme-linked immunosorbent assay and between-group differences (IM and NIM; NS and FS) were evaluated using the Wilcoxon signed-rank test. The association between pre-operative pain score and the level of each inflammatory mediator was evaluated using Spearman's correlation.

Results: Higher levels of TNF-a and IL-6 were identified in the IM tissue, compared to NIM ( $p<0.05)$. IL-6 levels were also higher in the NS compared to the FS $(p<0.05)$. There was no correlation between pre-operative pain score and level of each inflammatory mediator.

Conclusions: Our outcomes confirm a local increase in inflammatory mediator levels, in both meniscal and synovial tissue, which could contribute to development of OA. Management of these biological effects of meniscal injury might be warranted.
\end{abstract}

Keywords: Meniscal injury, Inflammatory mediator, Synovium, Osteoarthritis

\section{Background}

The menisci of the knee contribute significantly to the biomechanics of the knee joint, being important for load transmission, optimizing bone-on-bone contact and gliding movements, as well as working with the ligaments of the knee, including the anterior cruciate ligament, to provide joint stability (Kennedy et al. 1982; Seedhom et al. 1974). In view of the structural and functional importance

\footnotetext{
* Correspondence: togura@fffor.jp

${ }^{1}$ Funabashi Orthopaedic Hospital Sports Medicine Center, Funabashi, Chiba, Japan

Full list of author information is available at the end of the article
}

of the menisci, surgical management of meniscal injuries, which have become very common among professional and amateur athletes, is recommended and are in fact among the most common indications for knee surgery (Sihvonen et al. 2013).

Different studies have reported meniscal injury to be a risk factor for the development of knee joint osteoarthritis (OA) (Englund et al. 2009; Ding et al. 2007; Zeichen et al. 2006). The association between meniscal injury or meniscectomy and knee OA has largely been investigated from a biomechanics perspective, that is, how altered stability and joint loading contribute to the disease

\section{Springer}


process of OA. However, the biological effects of meniscal injury on the tissues of the knee joint remain unclear. In order to develop optimal therapeutic strategies, both the mechanical and biological pathways linking meniscal injury to knee OA must be understood.

The role of several inflammatory mediators in the disease process of knee OA has been studied, including tumor necrosis factor-alpha (TNF- $\alpha$ ), interleukin-6 (IL-6) and nerve growth factors (NGF) (Fiorito et al. 2005; Pearle et al. 2007; Smith et al. 1997). For example, high concentration of TNF- $\alpha$ and IL- 6 in synovial fluid were associated with early $\mathrm{OA}$ in an animal study (Venn et al. 1993). TNF- $\alpha$ can induce synovial cells and chondrocytes to produce IL-6, IL-8 and leukocyte inhibitory factor, as well as stimulate protease and prostaglandin production (Pelletier et al. 2001; Fernandes et al. 2002). High serum level of IL-6 has been reported to be a significant predictor of radiographic evidence of knee OA (Livshits et al. 2009). In addition, NGF was reported to be upregulated in chondrocytes and synovial fibroblasts in human $\mathrm{OA}$ joints, suggesting its crucial role in the pathology of OA (Iannone et al. 2002; Manni et al. 2003). Despite the important effect of these mediators on OA, the specific effect of inflammatory mediators associated to meniscal tears on meniscal and synovial tissue has yet to be clearly determined. In this study, we tested the hypothesis that injury to the meniscus would produce a local inflammatory response, involving both the meniscus and adjacent synovium, which could contribute to the development of OA. This hypothesis was evaluated by comparing levels of specific inflammatory mediators, TNF- $\alpha$, IL- 6 , and NGF, in injured and uninjured meniscal tissue and related knee joint synovium.

\section{Methods}

\section{Selection of the study group}

Prospective participants were patients who were evaluated at our hospital for a history of 'knee pain', between November 2011 and May 2012, and who were diagnosed as having sustained a lesion in the avascular zone of the medial or lateral meniscus of the knee. The diagnosis was formed from the medical history, physical examination and magnetic resonance imaging (MRI). Prospective participants were further assessed to exclude concomitant ligament deficiency, knee joint OA and injury to the articular cartilage. OA and articular cartilage injury were excluded on MRI preoperatively and by arthroscopy intraoperatively. Nineteen patients met our inclusion and exclusion criteria, provided informed consent, and were enrolled in the study. These patients subsequently underwent arthroscopic partial meniscectomy. The methods and procedures were approved by our institutional review board.

\section{Pain evaluation}

Prior to arthroscopy, patients were asked to rate the level of their knee pain, both at rest and during movement, on a 10-point numerical rating scale (NRS), with a score of ' 0 ' indicating 'no pain' and a score of '10' indicating the 'worst pain imaginable'.

\section{Arthroscopic procedure}

The injured meniscal tissue was resected using an oval punch, as per routine procedures for arthroscopic partial meniscectomy. Additional meniscal tissue around the lesion was resected to obtain a smooth meniscal surface. During the arthroscopic procedure, we differentiated injured and uninjured meniscal tissue. Meniscal tissue from two separate areas were resected and classified into two groups for analysis: injured tissue from the meniscal site (IM), which included the meniscal lesions, and meniscal tissue from the non-injured site (NIM), which did not include tissue from the site of the meniscal lesion (Fig. 1). Both IM and NIM were obtained from only the avascular zone of the meniscus. Samples of the synovium were obtained from two sites for analysis: the 'nearest' synovium (NS) was attached to the joint capsule near the site of the meniscal injury, and the 'farthest synovium' (FS) was obtained from the opposite joint

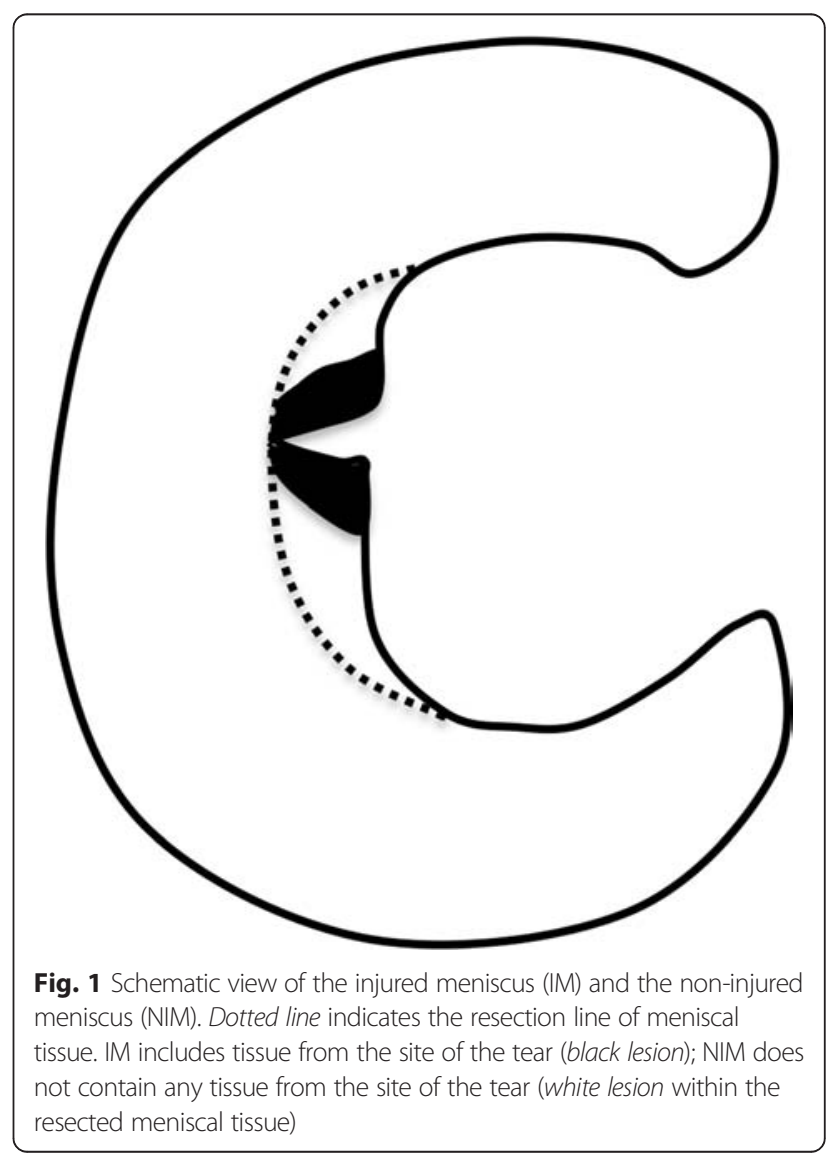


compartment (i.e., if the injury was to the medial meniscus, the synovium was obtained from the lateral compartment of the knee). Each sample included at least two or three pieces of tissue obtained using an oval punch.

Meniscal and synovial tissue samples were immediately stored at $-80{ }^{\circ} \mathrm{C}$. In preparation for analysis, the samples were thawed and subsequently diced and lysed with $500 \mu \mathrm{L}$ of CelLytic M Cell Lysis Reagent. Inflammatory mediator quantification was performed using a double-antibody sandwich enzyme-linked immunosorbent assays (ELISA) for TNF- $\alpha$, IL-6 (R\&D systems, Minneapolis, MN) and NGF (Boster Biological Tec, Wuhan, China), using the manufacturers' protocols. Tissue protein was assayed using the Bio-Rad kit (Bio-Rad Laboratories, Hercules, CA), again using the manufacturer's protocols. Levels of inflammatory mediators were normalized to the protein level of each tissue (Jonas et al. 2015; Miyagi et al. 2011).

\section{Statistical analysis}

Descriptive statistics were calculated for each measured variable. Differences in concentration of inflammatory mediators (TNF- $\alpha$, IL- 6 and NGF) between IM and NIM tissue samples, as well as between NS and FS, were evaluated using the Wilcoxon signed-rank test. A subanalysis was performed by sex and delay from the time of injury to surgery: acute group, less than 1 month from injury to surgery, and chronic group, more than 1 month from injury to surgery. The correlation between NRS score and the level of each inflammatory cytokine was evaluated using Spearman's rank correlation. The level of significance was set at $p<0.05$, a priori. All statistical analyses were performed with Stata (version 13; StataCorp LP, College Station, TX).

\section{Results}

\section{Description of the study group}

The study group consisted of 12 men and 7 women, with a mean age of $31.1 \pm 13.6$ years. The mean time to surgery after injury was 7.7 months, with a range of 2 weeks to 3 years. In this group, 5 patients underwent surgery less than 1 month after injury, forming the acute group (mean delay, 3.2 weeks; range, 2 weeks to 1 month), and the other 14 patients more than 1 month after injury, forming the chronic group (mean delay, 10.1 months; range, 2 month to 3 years). With regards to the NRS score, 7 patients reported pain at rest, with a mean NRS score of $1.8 \pm 2.3$, and all patients reported pain on movement, with a mean NRS score of $6.5 \pm$ 2.0 (Table 1).

\section{Concentrations of inflammatory mediators in the meniscus} Compared to the NIM tissue, TNF- $\alpha$ levels were elevated in the IM tissue IM, $0.16 \pm 0.052 \mathrm{pg} / \mathrm{mg}$; NIM, $0.048 \pm$

Table 1 Relevant demographic features of our study group

\begin{tabular}{|c|c|c|c|c|c|c|c|}
\hline Case & Age & Sex & BMI & $\begin{array}{l}\text { Side of } \\
\text { meniscus }\end{array}$ & $\begin{array}{l}\text { Mean time to } \\
\text { surgery (months) }\end{array}$ & $\begin{array}{l}\text { Tear } \\
\text { pattern }\end{array}$ & $\begin{array}{l}\text { NRS } \\
\text { At rest/during movement }\end{array}$ \\
\hline 1 & 15 & f & 20.3 & Lateral & 2 & Radial & $0 / 8$ \\
\hline 2 & 40 & $\mathrm{~m}$ & 26.6 & Medial & 12 & Horizontal & $6 / 5$ \\
\hline 3 & 37 & $\mathrm{~m}$ & 26.8 & Medial & 4 & Radial & $0 / 5$ \\
\hline 4 & 18 & $\mathrm{~m}$ & 20.2 & Lateral & 1 & Radial & $0 / 5$ \\
\hline 5 & 16 & $f$ & 19.3 & Lateral & 3 & Radial & $6 / 7$ \\
\hline 6 & 44 & $f$ & 21.9 & Lateral & 36 & Radial & $0 / 5$ \\
\hline 7 & 18 & $\mathrm{~m}$ & 20.8 & Lateral & 1 & Radial & $0 / 7$ \\
\hline 8 & 41 & $\mathrm{~m}$ & 24.1 & Medial & 0.5 & Horizontal & $0 / 5$ \\
\hline 9 & 39 & $\mathrm{~m}$ & 22.8 & Lateral & 2 & Radial & $0 / 4$ \\
\hline 10 & 32 & $\mathrm{~m}$ & 21.0 & Medial & 15 & Horizontal & $8 / 8$ \\
\hline 11 & 37 & $\mathrm{~m}$ & 19.5 & Lateral & 4 & Radial & $0 / 10$ \\
\hline 12 & 23 & f & 21.7 & Lateral & 7 & Radial & $4 / 5$ \\
\hline 13 & 20 & $\mathrm{~m}$ & 24.1 & Lateral & 2 & Radial & $0 / 7$ \\
\hline 14 & 35 & $\mathrm{~m}$ & 21.5 & Lateral & 24 & Radial & $0 / 3$ \\
\hline 15 & 33 & f & 28.9 & Medial & 1 & Horizontal & $3 / 6$ \\
\hline 16 & 59 & $\mathrm{~m}$ & 25.8 & Medial & 5 & Radial & $2 / 8$ \\
\hline 17 & 54 & $f$ & 25.2 & Medial & 24 & Radial & $0 / 8$ \\
\hline 18 & 17 & $\mathrm{~m}$ & 25.9 & Lateral & 2 & Radial & $0 / 8$ \\
\hline 19 & 13 & $f$ & 18.4 & Lateral & 0.5 & Radial & $6 / 10$ \\
\hline
\end{tabular}


$0.0096 \mathrm{pg} / \mathrm{mg} ; p=0.028$; Fig. 2a). IL-6 levels were also elevated in the IM tissue, compared to NIM tissue (IM, $0.11 \pm$ $0.014 \mathrm{pg} / \mathrm{mg}$; NIM, $0.067 \pm 0.0099$ pg/mg; $p=0.038$; Fig. $2 \mathrm{~b}$ ). Levels of NGF were comparable between the two meniscal tissues (IM, $0.044 \pm 0.0042$; NIM, $0.042 \pm 0.0040 \mathrm{pg} / \mathrm{mg}$; $p=0.31$; Fig. 2c).

\section{Concentrations of inflammatory mediators in the synovium} IL-6 levels were elevated in the NS synovial tissue, compared to the FS (NS, $0.088 \pm 0.0015 \mathrm{pg} / \mathrm{mg}$; FS, $0.076 \pm$ $0.0057 \mathrm{pg} / \mathrm{mg} ; p=0.039$; Fig. 3b). Levels of TNF- $\alpha$ and NGF were comparable in both NS and FS tissues (Fig. 3a, c).

\section{Correlation between NRS and levels of inflammatory mediators}

The correlation between NRS and level of each inflammatory mediator is reported in Table 2 . No correlation was identified between these parameters.

\section{Subanalysis}

Results of our subanalysis are reported in Tables 3 and 4 . No significant between-group or between-sex differences were identified.

\section{Discussion}

The development of knee OA after meniscal injury is a significant health problem, leading to pain and limitations in activities and participation. Although the contribution of effects of altered knee mechanics, resulting from meniscal injury and meniscectomy, on the development of OA have been investigated, the effects of associated biological factors on the disease process of OA following injury have not been addressed. To this end, we investigated the difference in inflammatory mediator levels, perioperatively, in injured and non-injured meniscal tissue, as well as in the associated synovial tissue. Our study demonstrated a significant elevation of TNF$\alpha$ and IL- 6 cytokines in the injured meniscal tissue, with a concomitant increase of IL-6 in the synovial tissue local to the site of injury. This is an important finding as inflammatory mediators have been reported to play an important role in the development of knee OA (Edd et al. 2015; Olson et al. 2014). TNF- $\alpha$ directly stimulates sensory neurons, via its receptors, which initiates a cascade of inflammatory responses mediated through the production of ILs, including IL-1 and IL-6 (Ohtori et al. 2004; Pollock et al. 2002). In particular, there is evidence for a complex role of IL- 6 in the pathogenesis of OA, mediated specifically through its effect on the production of tissue inhibitors of metalloproteinases-1 (MMP-1), which might influence the extent of cartilage damage, post-injury, through negative feedback (Lotz and Guerne. 1991). Thus, our identification of an elevation in TNF- $\alpha$ and IL- 6 at the site of injury is indicative of a local inflammatory response which could initiate the cycle of cartilage damage leading to the development of early knee OA in the area local to the injury. As our intent was to clarify differences in inflammatory mediators induced by the injury, we obtained and evaluated tissue samples from the avascular zone of the meniscus. Although our findings cannot be extrapolated to tissue from the vascular zone of the meniscus, our use of avascular tissue prevented possible blood contamination, which would have made accurate comparison difficult.

Previous studies have reported elevations of TNF- $\alpha$ and IL- 6 in the joint fluid after acute knee injury, including injury to the meniscus and to the anterior cruciate ligament (Tang et al. 2009; Higuchi et al. 2006; Irie et al. 2003). Although we evaluated inflammatory responses specifically in meniscal and synovial tissue, findings from these previous studies are consistent with ours. It is possible that inflammatory mediators produced by injured meniscal tissue could be one of the origins contributing to a general inflammatory response of the knee joint after meniscal injuries. Age may be an important factor influencing the inflammatory response of tissues following meniscal injury. Specifically, Brophy et al. have recently
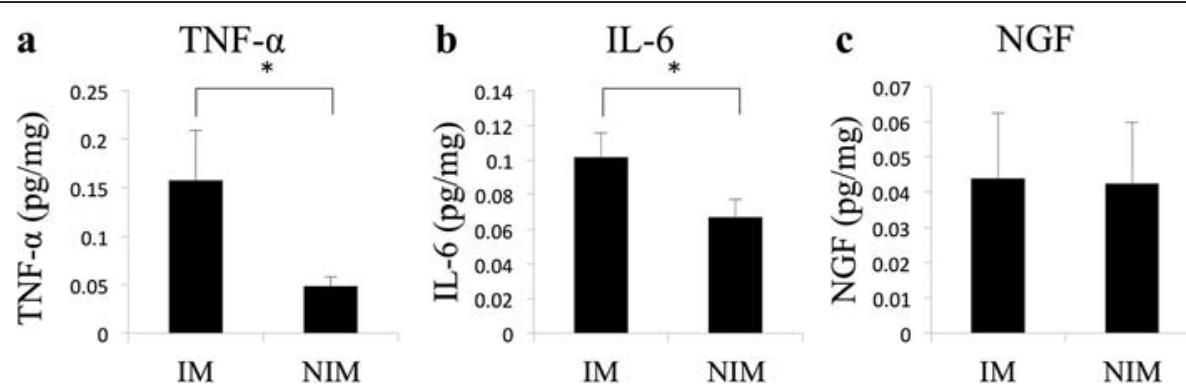

Fig. 2 Levels of inflammatory mediators in meniscal tissues a tumor necrosis factor-alpha (TNF-a) and $\mathbf{b}$ interleukin-6 (IL-6), showing the significant elevation of levels in injured meniscal tissue (IM), compared to levels in non-injured tissue (NIM); c Comparable levels of nerve growth factor (NGF) in both IM and NIM tissues is shown; values are reported as a mean \pm SD; ${ }^{*} p<0.05$ 

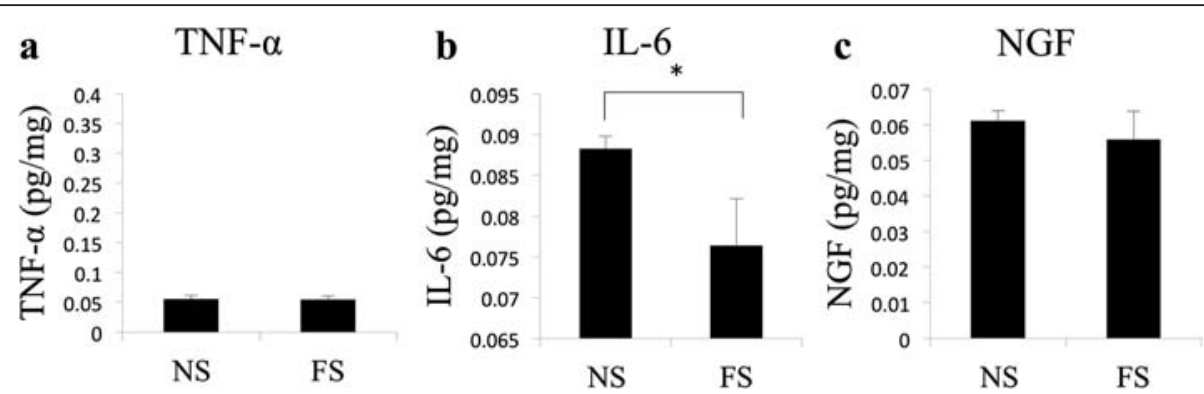

Fig. 3 Concentrations of inflammatory mediators in the synovium, a showing the significant elevation of interleukin-6 level in the nearest synovial tissue (NS); levels of $\mathbf{b}$ tumor necrosis factor-alpha (TNF-a) and $\mathbf{c}$ nerve growth factor (NGF) are comparable in IM and NIM tissues; values are reported as a mean $\pm \mathrm{SD} ;{ }^{*} p<0.05$

reported an elevation in the gene expression of arthritisrelated markers, including a disintegrin and metalloproteinase with thrombospondin motifs 5 (ADAMTS-5), MMP-1 and MMP-13 in patients with meniscal injuries under the age of 40 years (Brophy et al. 2012). These findings provide evidence of clinically relevant, age-dependent differences in the response of the knee to meniscal tears, with elevated expression of arthritis-related markers, indicative of an increased catabolic response, in patients under 40 years of age. Future research is required to evaluate differences in gene expression of arthritis markers in injured and non-injured meniscal tissue to fully characterize the role of genetic factors in the disease pathway relating meniscal injuries to early onset of knee OA. Our findings demonstrating significant elevation of inflammatory mediators in injured and uninjured meniscal tissue, and in the synovium local to the injury, provide justification for future studies to clarify the role of this local inflammatory response of damaged meniscal tissue on the development of OA.

We did not identify a correlation between preoperative NRS scores and levels of the different inflammatory mediators evaluated. A relationship between the level of inflammatory mediators and patient-reported pain scores have, however, been reported by other researchers. Cuellar et al. reported a correlation between levels of IL6 in synovial fluid and pain score in patients with meniscal tear, whereas there was no evidence of an effect of levels of TNF- $\alpha$ (Cuellar et al. 2009). Similarly, Cuellar et al. reported a correlation between levels of IL-6 in

Table 2 Correlation between Pre-operative NRS and Levels of Inflammatory Mediators

\begin{tabular}{|c|c|c|c|c|c|c|c|}
\hline & \multicolumn{2}{|c|}{ TNF-a } & \multicolumn{2}{|l|}{ IL-6 } & \multirow{2}{*}{$\begin{array}{l}\text { NGF } \\
Y \\
\end{array}$} & \multirow[b]{2}{*}{$p$-value } \\
\hline & & $\gamma$ & $p$-value & Y & $p$-value & & \\
\hline \multirow[t]{2}{*}{ NRS } & Meniscus & 0.3 & 0.21 & 0.36 & 0.13 & -0.19 & 0.25 \\
\hline & Synovium & 0.046 & 0.86 & -0.029 & 0.91 & -0.31 & 0.74 \\
\hline
\end{tabular}

NRS numerical rating scale, TNF- $a$ tumor necrosis factor-alpha, IL-6 interleukin-6, NGF nerve growth factor synovial fluid and pain score in patients with tears of the anterior cruciate ligament, with no effect associated to TNF- $\alpha$ levels (Cuellar et al. 2010). In contrast, Orita et al. reported a positive correlation between level of TNF$\alpha$ in synovial fluid and pain score in patients with OA, with no effect of levels of IL-6 (Orita et al. 2011). Interestingly, among patients undergoing total knee replacement, a two-fold increase from baseline in plasma IL-6 levels was reported, post-surgery, which had no correlation to knee pain scores (Feng et al. 2008). Therefore, it is possible that the correlation between levels of inflammatory mediators and patient reported pain may be disease-dependent, which might explain the absence of a positive correlation in our study. The absence of a correlation could also result from the absence of sensory nerve endings in our sample meniscal tissues, which were obtained from the avascular zone of the meniscus. Previous study found that nerve fibers and sensory receptors mainly exist in the periphery and vascular zone of the meniscus, representing the outer one-third of the meniscus (Day et al. 1985). In addition, we did not identify an elevation of NGF level in the injured meniscal tissue, an inflammatory cytokine that specifically stimulates sensory nerve growth. The absence of an influence of meniscal injury on NGF could further explain the absence of sensory nerve growth in the injured tissue. Future studies on the association between meniscal injury, inflammatory response, and pain should consider the role of other molecules, such as prostaglandins, cyclooxygenase, and nitric oxide.

We also did not identify a correlation between elevated IL- 6 in the synovium membrane and knee pain scores. This finding is corroborated by that of Scanzello et al.'s study indicating no correlation between the absence or presence of synovitis and preoperative pain scores (Scanzello et al. 2011). However, Scanzello et al. did report a positive correlation between presence of joint synovitis and functional score on the Lysholm scale. Therefore, it is likely that inflammation of the synovium affects knee function to a greater extent than 
Table 3 Level of inflammatory mediators in acute and chronic group

\begin{tabular}{|c|c|c|c|c|c|c|c|}
\hline & & \multicolumn{3}{|c|}{ Meniscus (pg/mg) } & \multicolumn{3}{|c|}{ Synovium (pg/mg) } \\
\hline & & $\mathrm{IM}$ & NIM & $p$-value & NS & FS & $p$-value \\
\hline & & Mean & Mean & & & & \\
\hline \multirow[t]{2}{*}{$\overline{\text { TNF-a }}$} & Acute & $0.44 \pm .14$ & $0.044 \pm .007$ & .043 & $0.055 \pm .004$ & $0.053 \pm 0.053$ & n.s. \\
\hline & Chronic & $0.059 \pm .042$ & $0.049 \pm .022$ & .03 & $0.055 \pm .006$ & $0.055 \pm .006$ & n.s. \\
\hline \multirow[t]{2}{*}{ IL-6 } & Acute & $0.12 \pm .03$ & $0.055 \pm .014$ & .043 & $0.087 \pm .02$ & $0.051 \pm .14$ & .043 \\
\hline & Chronic & $0.096 \pm .017$ & $0.071 \pm .012$ & .03 & $0.088 \pm .007$ & $0.082 \pm .014$ & .015 \\
\hline \multirow[t]{2}{*}{ NGF } & Acute & $0.049 \pm .082$ & $0.042 \pm .084$ & n.s. & $0.053 \pm .01$ & $0.061 \pm .017$ & n.s. \\
\hline & Chronic & $0.042 \pm .004$ & $0.042 \pm .005$ & n.s. & $0.057 \pm .02$ & $0.061 \pm .03$ & n.s. \\
\hline
\end{tabular}

TNF- $a$ tumor necrosis factor-alpha, IL-6 interleukin-6, NGF nerve growth factor, IM injured meniscal site, NIM non-injured meniscal site, NS synovium 'nearest' the lesion, FS 'farthest' synovium, n.s. not significantly different, SD standard deviation

pain. Future studies investigating the role of inflammation on knee pain and function should include scales of knee function, such as the Knee injury and Osteoarthritis Outcome Score (KOOS) and Lysholm scales.

Taken together, our results potentially indicate that a meniscal injury results in a significant elevation in the expression of the inflammatory mediators TNF-alpha and IL- 6 locally at the site of injury, and of IL-6, in the synovium in close proximity to the injury. These local inflammatory responses could initiate the process of articular cartilage degeneration. However, the limitations of our study need to be noted in the interpretation of our results. Foremost, although we separated meniscal tissue from injured and uninjured sites for analysis, it is important to recognize that all sampled were obtained from patients with meniscal injury. No published data were identified in healthy menisci for comparison. Also, a variety of other factors could influence the level of inflammatory mediators in meniscal tissue, such as general health conditions, body mass index, smoking status, and age. Finally, extracellular signaling molecules are sometimes found attached to the extracellular matrix in injured joints. Therefore, it is possible that the cellular origins of inflammatory mediators may not be detectable by ELISA. In order to confirm whether these molecules are produced by the meniscus and synovium, quantitative real-time reverse transcription polymerase chain reaction (RT-PCR) analysis will be needed. However, we note the presence of RNA may not always accurately reflect tissue protein levels, which is a major disadvantage of RT-PCR analysis and why we used ELISA in our study, as it allowed us to identify secreted cytokines at the protein level.

\section{Conclusions}

Our findings provide Level III evidence of a specific role of local inflammatory mediators in the disease process of meniscal lesions, which might contribute to the development of knee OA. Specifically, levels of inflammatory mediators TNF- $\alpha$ and IL- 6 increased significantly at the site of the meniscal lesion, and IL- 6 levels in the local synovium. Based on these results, we propose that management of these biological effects of meniscal injury might be warranted. However, future studies are required to evaluate the possible interaction between these biological effects on the mechanical pathway for the development of knee OA after meniscal injuries.

Table 4 Level of inflammatory mediators in male and female

\begin{tabular}{|c|c|c|c|c|c|c|c|}
\hline & & \multicolumn{3}{|c|}{ Meniscus (pg/mg) } & \multicolumn{3}{|c|}{ Synovium (pg/mg) } \\
\hline & & $\mathrm{IM}$ & NIM & $p$-value & NS & FS & $p$-value \\
\hline & & Mean \pm SD & Mean \pm SD & & Mean \pm SD & Mean \pm SD & \\
\hline \multirow[t]{2}{*}{ TNF-a } & Male & $0.12 \pm .053$ & $0.048 \pm .03$ & .018 & $0.055 \pm .002$ & $0.055 \pm .002$ & n.s. \\
\hline & Female & $0.23 \pm .11$ & $0.046 \pm .004$ & .04 & $0.054 \pm .002$ & $0.053 \pm .002$ & n.s. \\
\hline \multirow[t]{2}{*}{ |L-6 } & Male & $0.079 \pm .069$ & $0.068 \pm .014$ & .049 & $0.088 \pm .0021$ & $0.075 \pm .0072$ & .003 \\
\hline & Female & $0.14 \pm .034$ & $0.065 \pm .012$ & .018 & $0.089 \pm .0034$ & $0.072 \pm .011$ & .018 \\
\hline \multirow[t]{2}{*}{ NGF } & Male & $0.041 \pm .005$ & $0.041 \pm .006$ & n.s. & $0.061 \pm .001$ & $0.054 \pm .003$ & n.s. \\
\hline & Female & $0.044 \pm .0072$ & $0.048 \pm .0067$ & n.s. & $0.062 \pm .012$ & $0.06 \pm .02$ & n.s. \\
\hline
\end{tabular}

TNF- $a$ tumor necrosis factor-alpha, IL-6 interleukin-6, NGF nerve growth factor, IM injured meniscal site, NIM non-injured meniscal site, NS synovium 'nearest' the lesion, FS 'farthest' synovium, n.s. not significantly different, SD standard deviation 


\section{Abbreviations}

OA: osteoarthritis; TNF-a: tumor necrosis factor-alpha; IL-6: interleukin-6; NGF: nerve growth factor; IM: injured meniscal site; NIM: non-injured meniscal site; NS: synovium 'nearest' the lesion; FS: 'farthest' synovium; NRS: numerical rating scale; ELISA: enzyme-linked immunosorbent assays; ADAMTS-5: a disintegrin and metalloproteinase with thrombospondin motifs 5; MMP: matrix metalloproteinase; RT-PCR: reverse transcription polymerase chain reaction.

\section{Competing interests}

The authors declare that they have no competing interests.

\section{Authors' contributions}

TO carried out all experiments, analyzed data and drafted the manuscript. YS, $\mathrm{KY}, \mathrm{SO}, \mathrm{MM}, \mathrm{TI}, \mathrm{HK}$ and $\mathrm{YO}$ participated in the design of the study. IK, KT, HS, $\mathrm{TN}$, and HF harvested meniscal tissue and synovial membrane, and participated in the design of the study. MS, KT, SO and AT conceived the study and participated in its design and coordination and helped to draft the manuscript. All authors read and approved the final manuscript.

\section{Acknowledgments}

All authors declare no funding sources or sponsors in this study.

\section{Author details}

${ }^{1}$ Funabashi Orthopaedic Hospital Sports Medicine Center, Funabashi, Chiba, Japan. ${ }^{2}$ Department of Orthopaedic Surgery, Graduate School of Medicine, Chiba University, Chiba, Japan.

\section{Received: 4 November 2015 Accepted: 25 January 2016} BPt?

\section{References}

Brophy RH, Rai MF, Zhang Z, Torgomyan A, Sandell LJ (2012) Molecular analysis of age and sex-related gene expression in meniscal tears with and without a concomitant anterior cruciate ligament tear. J Bone Joint Surg Am 94:385-93. doi:10.2106/JBJS.K.00919

Cuellar JM, Scuderi GJ, Cuellar VG, Golish SR, Yeomans DC (2009) Diagnostic utility of cytokine biomarkers in the evaluation of acute knee pain. J Bone Joint Surg Am 91:2313-2320. doi:10.2106/JBJS.H.00835

Cuellar VG, Cuellar JM, Golishh SR, Yeomans DC, Scuderi GJ (2010) Cytokine profiling in acute anterior cruciate ligament injury. Arthroscopy 26:1296-1301. doi:10.1016/j.arthro.2010.02.011

Day B, Mackenzie WG, Shim SS, Leung G (1985) The vascular and nerve supply of the human meniscus. Arthroscopy 1:58-62, PMID: 4091911

Ding C, Martel-Pelletier J, Pelletier JP, Abram F, Raynauld JP, Cicuttini F, Jones G (2007) Meniscal tear as an osteoarthritis risk factor in a largely non-osteoarthritic cohort: a cross-sectional study. J Rheumatol 34:776-784, PMID: 17361984

Edd SN, Giori NJ, Andriacchi TP (2015) The role of inflammation in the initiation of osteoarthritis after meniscal damage. J Biomech 48:1420-1426. doi:10.1016/j.jbiomech.2015.02.035

Englund M, Guermazi A, Lohmander LS (2009) The meniscus in knee osteoarthritis. Rheum Dis Clin North Am 35:579-590. doi:10.1016/j.rdc.2009.08.004

Feng Y, Ju H, Yang B, An H (2008) Effects of a selective cyclooxygenase-2 inhibitor on postoperative inflammatory reaction and pain after total knee replacement. J Pain 9:45-52. doi:10.1016/j.jpain.2007.08.003

Fernandes JC, Martel-Pelletier J, Pelletier JP (2002) The role of cytokines in osteoarthritis pathophysiology. Biorheology 39(1-2):237-46, PubMed

Fiorito S, Magrini L, Adrey J, Mailhé D, Brouty-Boyé D (2005) Inflammatory status and cartilage regenerative potential of synovial fibroblasts from patients with osteoarthritis and chondropathy. Rheumatology 44:164-171. doi:10.1093/rheumatology/keh431

Higuchi H, Shirakura K, Kimura M, Terauchi M, Shinozaki T, Watanabe H, Takagishi $\mathrm{K}$ (2006) Changes in bio-chemical parameters after anterior cruciate ligament injury. Int Orthop 30:43-47, PMID: 16333657

lannone F, De Bari C, Dell'Accio F, Covelli M, Patella V, Lo Bianco G, Lapadula G (2002) Increased expression of nerve growth factor (NGF) and high affinity NGF receptor (p140 TrkA) in human osteoarthritic chondrocytes. Rheumatology (Oxford) 41(12):1413-1418

Irie K, Uchiyama E, Iwaso H (2003) Intraarticular inflammatory cytokines in acute anterio cruciate ligament injured knee. Knee 10:93-96. doi:10.1016/S0968-0160(02)00083-2

Jonas Ml, Kurylowicz A, Bartoszewicz Z, Lisik W, Jonas M, Wierzbicki Z, Chmura A, Pruszczyk P, Puzianowska-Kuznicka M (2015) Interleukins 6 and 15 levels are higher in subcutaneous adipose tissue, but obesity is associated with their increased content in visceral fat depots. Int J Mol Sci 16(10):25817-30. doi:10.3390/ijms161025817, PubMed PMID: 26516848; PubMed Central PMCID: PMCPMC4632828

Kennedy JC, Alexander IJ, Hayes KL (1982) Nerve supply of the human knee and its functional importance. Am J Sports Med 10:329-335. doi:10.1177/036354658201000601

Livshits G, Zhai G, Hart DJ, Kato BS, Wang H, Williams FM et al (2009) Interleukin-6 is a significant predictor of radiographic knee osteoarthritis: The Chingford Study. Arthritis Rheum 60(7):2037-45. doi:10.1002/art.24598, PubMed PMID: 19565477; PubMed Central PMCID: PMCPMC2841820

Lotz M, Guerne PA (1991) Interleukin-6 induces the synthesis of tissue inhibitor of metalloproteinases-1/erythroid potentiating activity (TIMP-1/EPA). J Biol Chem 266:2017-2020, PMID: 1846608

Manni L, Lundeberg T, Fiorito S, Bonini S, Vigneti E, Aloe L (2003) Nerve growth factor release by human synovial fibroblasts prior to and following exposure to tumor necrosis factor-alpha, interleukin-1 beta and cholecystokinin-8: the possible role of NGF in the inflammatory response. Clin Exp Rheumatol 21(5):617-624, PubMed

Miyagi M, Ishikawa T, Orita S, Eguchi Y, Kamoda H, Arai G, Suzuki M, Inoue G, Aoki Y, Toyone T, Takahashi K, Ohtori S (2011) Disk injury in rats produces persistent increases in pain-related neuropeptides in dorsal root ganglia and spinal cord glia but only transient increases in inflammatory mediators: pathomechanism of chronic diskogenic low back pain. Spine (Phila Pa 1976) 36(26):2260-2266. doi:10.1097/BRS.0b013e31820e68c7

Ohtori S, Takahashi K, Moriya H, Myers RR (2004) TNF-a and TNF-a receptor type 1 upregulation in glia and neurons after peripheral nerve injury: studies in murine DRG and spinal cord. Spine 29:1082-1088, PMID: 15131433

Olson SA, Horne P, Furman B, Huebner J, Al-Rashid M, Kraus VB, Guilak F (2014) The role of cytokines in posttraumatic arthritis. J Am Acad Orthop Surg 22:29-37. doi:10.5435/JAAOS-22-01-29

Orita S, Koshi T, Mitsuka T, Miyagi M, Inoue G, Arai G, Ishikawa T, Hanaoka E, Yamashita K, Yamashita M, Eguchi Y, Toyone T, Takahashi K, Ohtori S (2011) Associations between proinflammatory cytokines in the synovial fluid and radiographic grading and pain related scores in 47 consecutive patients with osteoarthritis of the knee. BMC Musculoskelet Disord 12:144. doi:10.1186/1471-2474-12-144

Pearle AD, Scanzello CR, George S, Mandl LA, DiCarlo EF, Peterson M, Sculco TP, Crow MK (2007) Elevated high-sensitivity C-reactive protein levels are associated with local inflammatory findings in patients with osteoarthritis. Osteoarthr Cartilage 15:516-523. doi:10.1016/j.joca.2006.10.010

Pelletier JP, Martel-Pelletier J, Abramson SB (2001) Osteoarthritis, an inflammatory disease: potential implication for the selection of new therapeutic targets. Arthritis Rheum 44(6):1237-1247, PubMed PMID: 12082286

Pollock J, McFarlane SM, Connell MC, Zehavi U, Vandenabeele P, MacEwan DJ, Scott RH (2002) TNF-alpha receptors simultaneously activate Ca2+ mobilisation and stress kinases in cultured sensory neurons. Neuropharmacol 42:93-106. doi:10.1016/s0028-3908(01)00163-0

Scanzello CR, McKeon B, Swaim BH, DiCarlo E, Asomugha EU, Kanda V, Nair A Lee DM, Richmond JC, Katz JN, Crow MK, Goldring SR (2011) Synovial inflammation in patients undergoing arthroscopic meniscectomy: molecular characterization and relationship to symptoms. Arthritis Rheum 63:391-400. doi:10.1002/art.30137

Seedhom BB, Dowson D, Wright V (1974) Function of menisci: a preliminary study. J Bone Joint Surg Br 33:381-382. doi:10.1136/ard.33.1.111

Sihvonen R, Paavola M, Malmivaara A, Itälä A, Joukainen A, Nurmi H, Kalske J, Järvinen TLN, for the Finnish Degenerative Meniscal Lesion Study (FIDELITY) Group (2013) Arthroscopic partial meniscectomy vs sham surgery for a degenerative meniscal tear. N Engl J Med 369:2515-24. doi:10.1056/NEJMoa1305189

Smith MD, Triantafillou S, Parker A, Youssef PP, Coleman M (1997) Synovial membrane inflammation and cytokine production in patients with early osteoarthritis. J Rheumatol 24:365-371, PMID: 9034998

Tang Z, Yang L, Wang Y, Xue R, Zhang J, Huang W, Chen PC, Sung KL (2009) Contributions of different intraarticular tissues to the acute phase elevation of synovial fluid MMP-2 following rat ACL rupture. J Orthop Res 27:243-248. doi:10.1002/jor.20763

Venn G, Nietfeld JJ, Duits AJ, Brennan FM, Arner E, Convington M, Billingham ME, Hardingham TE (1993) Elevated synovial fluid levels of interleukin-6 and tumor necrosis factor associated with early experimental canine osteoarthritis. Arthritis Rheum 36:819-26. PubMed

Zeichen J, Hankemeier S, Knobloch K, Jagodzinski M (2006) Arthroscopic partial meniscectomy. Oper Orthop Traumatol 18:380-392, PMID: 17171326 